Article

\title{
Plasma Deflection Interrupter for Pulsed Power Applications
}

\author{
Nathan Majernik, Walter Lynn, Yusuke Sakai and James Rosenzweig * \\ Department of Physics and Astronomy, University of California Los Angeles, Los Angeles, CA 90095, USA; \\ NMajernik@g.ucla.edu (N.M.); walter.j.lynn@gmail.com (W.L.); yusuke@physics.ucla.edu (Y.S.) \\ * Correspondence: rosen@physics.ucla.edu; Tel.: +1-310-206-4541
}

Received: 1 June 2018; Accepted: 27 July 2018; Published: 29 July 2018

\begin{abstract}
A plasma-based opening switch, capable of handling kiloampere currents at kilovolt potentials on the microsecond timescale, is described and characterized. The principle of operation is the deflection of a laser-induced arc by an external magnetic field to a shunt circuit path. A first-order model of operation is introduced. Finally, the merits of the device, particularly when applied to high-repetition-rate dense plasma focus (DPF) systems, are discussed.
\end{abstract}

Keywords: plasma; pulsed power; opening switch

\section{Introduction}

Opening switches for pulsed power applications, capable of holding off kilovolt potentials and conducting kiloampere currents, tend to be: fast ( $\ll$ millisecond switching), simple and robust; or inexpensive, but do not simultaneously possess all three traits. Common solutions for such applications include spark gaps (including magnetic [1], mechanical [2], and gas quenched [3,4] variants), plasma erosion opening switches (PEOS) [5,6], and semiconductor switches [7]. A summary of opening switch technologies is shown in Figure 1. We presented a fast, simple, and inexpensive opening switch, based on the deflection of an arc by an external magnetic field (Figure 2) intended to interrupt the current flow to a load. The arc is initialized between the input and load by laser breakdown, before being deflected by an applied magnetic field and then preferentially conducting a current through a shunt path. Unlike quenched spark gaps which rely on relatively slow processes, such as mechanical motion, gas flow, or heat transfer, this device operates on the microsecond timescale, due to its reliance on the motion of a plasma, which is accelerated at rates in excess of $10^{6} \mathrm{~m} / \mathrm{s}^{2}$. 


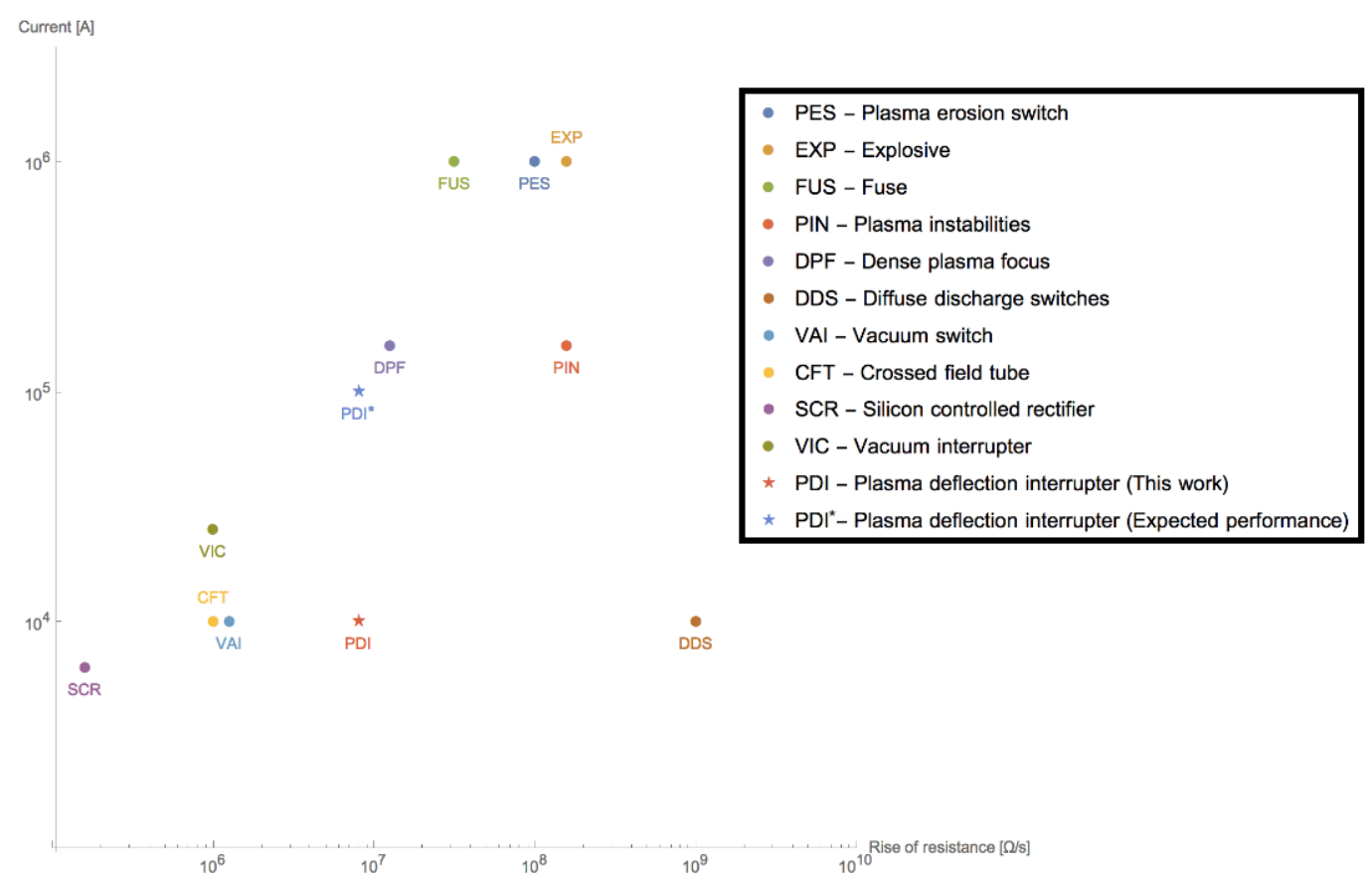

Figure 1. Summary of pulsed power opening switches, adapted from [8]. This current work has been included.

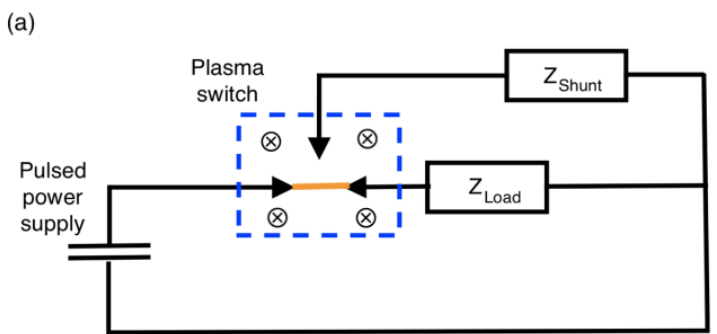

(b)
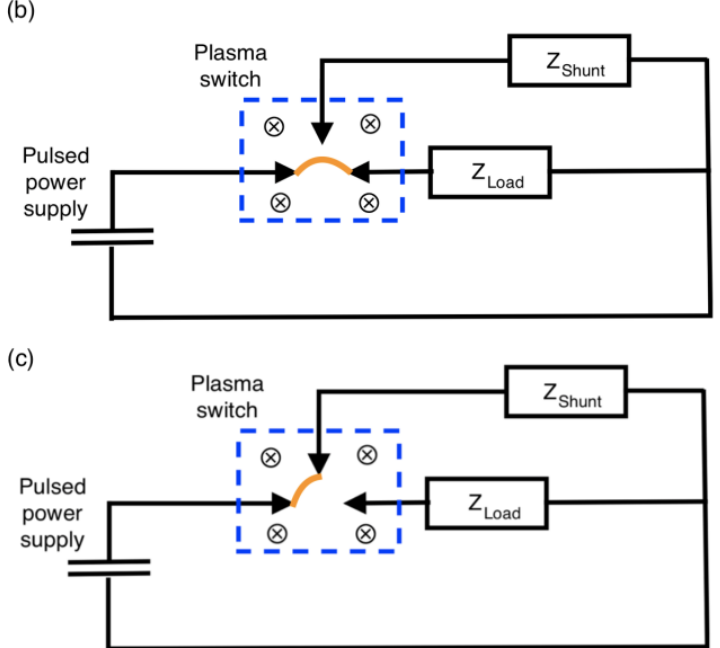

Figure 2. Schematic of switch operation. (a) Laser-induced breakdown of an arc between the supply and load occurs, (b) the external magnetic field increasingly deflects the arc, and (c) the arc begins preferentially conducting a current through the shunt. 


\section{Materials and Methods}

The device was constructed from UHMW plastic, copper bus bars, brass screws, and neodymium permanent magnets (Figure 3). The spacing between the load, shunt, and input electrodes were all independently variable. An annotated cross section of the switch is shown in Figure 4. The permanent magnets provided a 0.6-T magnetic field. The Nd:YAG triggered laser pulse had 500-mJ energy and 5-ns duration. The pulsed power supply was a 312-nF oil-filled capacitor, charged between 10 and 20 $\mathrm{kV}$. The load circuit had a total resistance of $0.12 \Omega$ and an inductance of $1440 \mathrm{nH}$. The shunt circuit had a total resistance of $0.09 \Omega$ and an inductance of $700 \mathrm{nH}$. The time derivative of the current $\frac{d I}{d t}$ in each circuit was monitored using custom $B$-dot probes.

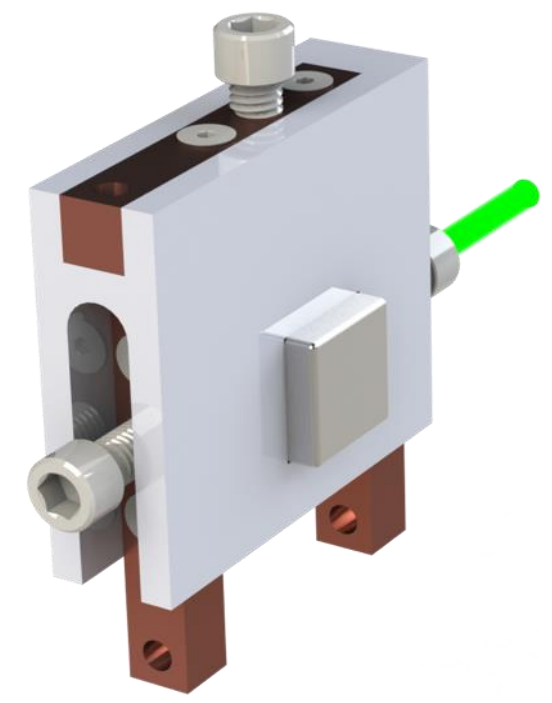

Figure 3. Render of a plasma switch.

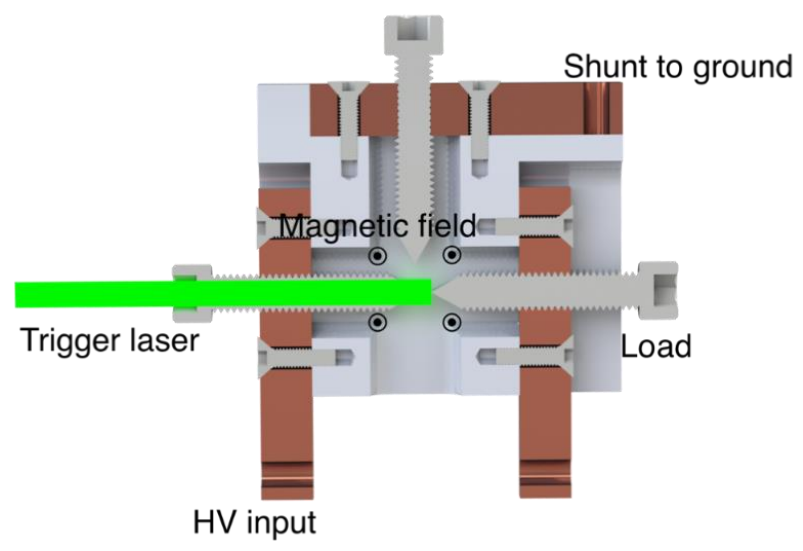

Figure 4. Cross section of a plasma switch.

\section{Results}

\subsection{Basic Operation}

Figure 5 shows the impact of varying the distance between the tip of the shunt electrode and the centerline of the input and load electrodes. In the first frame, with the shunt at $14 \mathrm{~mm}$ from the centerline, no current handoff occurred and the plasma switch operated like a typical laser triggered spark gap, which was the same as the behavior exhibited when the magnets were removed. In the second frame, the shunt electrode was moved in to $12 \mathrm{~mm}$, and after about $20 \mu \mathrm{s}$ (5 periods), handoff of 
current to the shunt path occurs. The apparent non-constant nature of $\frac{d I}{d t}$ and the observed frequency change were due to the lower impedance of the shunt path. By adjusting the shunt even closer in the third frame, e.g., to $10 \mathrm{~mm}$, handoff occurred in a shorter period, e.g., only after $15 \mu \mathrm{s}$. Finally, in the last frame, the shunt was the preferred path from the beginning of conduction.

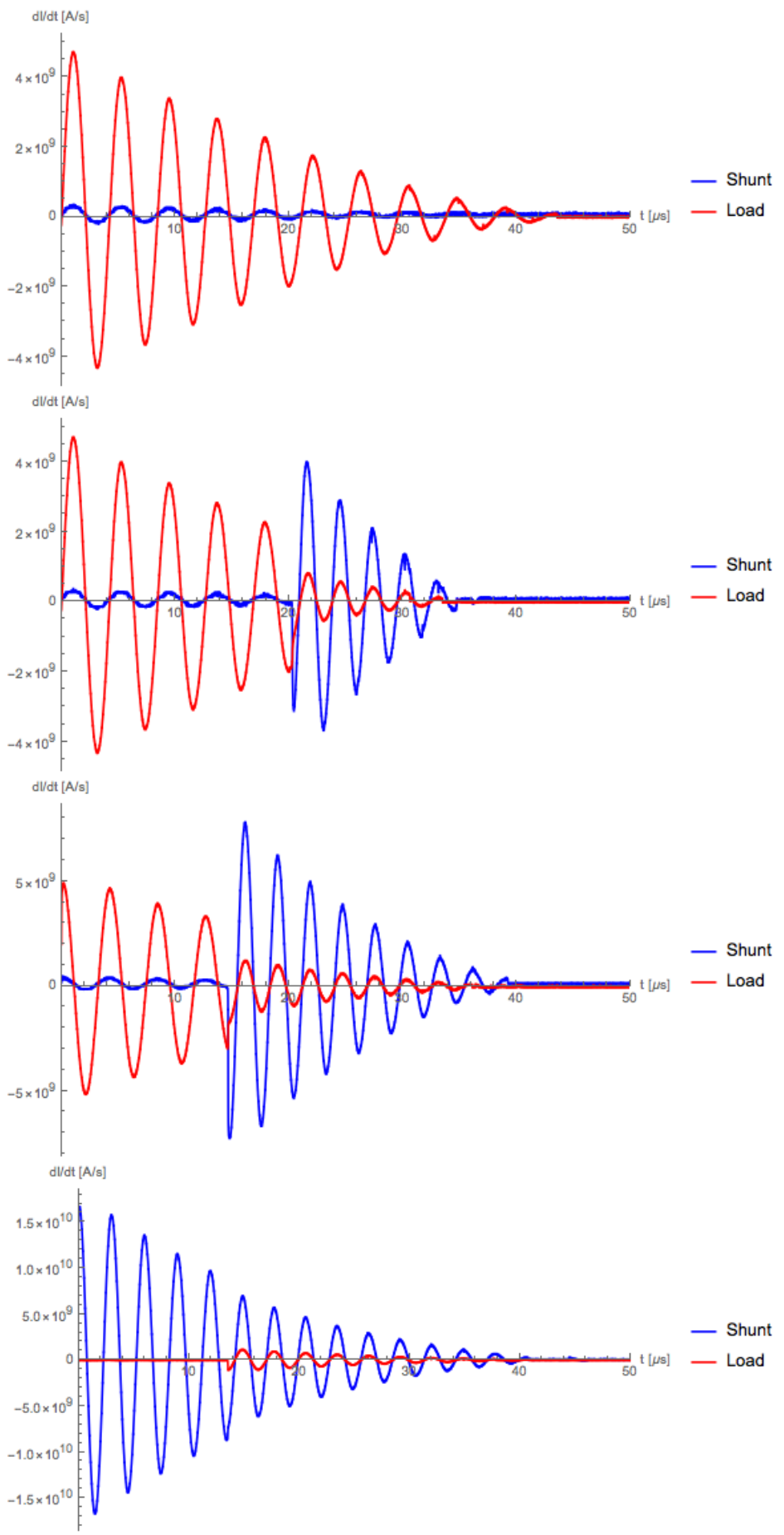

Figure 5. dI/dt traces with shunt-to-centerline distances of $14 \mathrm{~mm}, 12 \mathrm{~mm}, 10 \mathrm{~mm}$, and 8 $\mathrm{mm}$, respectively. 


\subsection{Analysis}

Plasma switches have a rich history including theoretical models for their operation, as is presented in e.g., Ref. [9]. However, the microscopic aspects of such models are beyond the scope of this article, and we satisfied ourselves with a phenomenological and approximate approach to characterize the behavior of this instrument, while omitting the details of the actual plasma dynamics. This simplified approach is based on the magnetic force (per unit volume) $|\boldsymbol{J} \times \boldsymbol{B}|$, and a decaying sinusoidal current flow $I(t) \propto V e^{-t \alpha} \sin (t \omega)$, where $V$ is the voltage, $\alpha$ is the decay constant, and $\omega$ is the RLC frequency. The simulated transverse positions of the arc are shown in Figure 6 for three different voltages: $V_{\text {green }}=2 V_{\text {blue }}, V_{\text {orange }}=1.5 V_{\text {blue }}$. The linear approximations of the motion for each case, where the slope is proportional to the voltage, are shown in Figure 6.

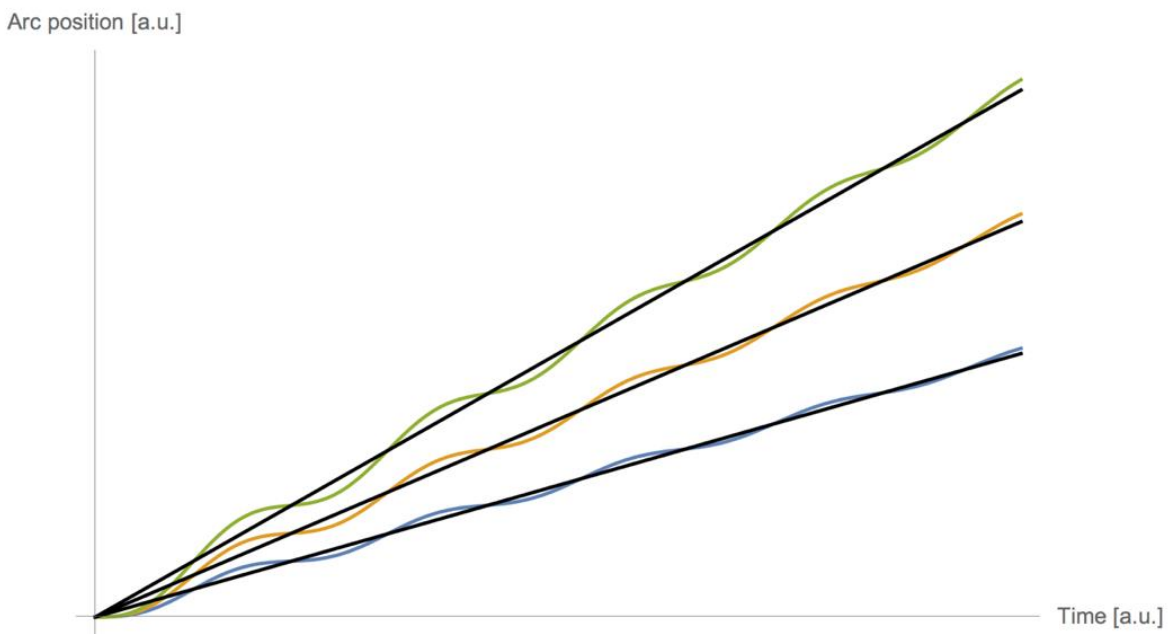

Figure 6. Transverse arc positions for different voltages $\left(V_{\text {green }}=2 V_{\text {blue }}, V_{\text {orange }}=1.5 V_{\text {blue }}\right)$. The linear approximation for each linear approximation is shown in black.

As long as the duration is longer than a few periods, a linear approximation is accurate. However, the initial conditions must also be considered; in Figure 6, the arc position was assumed to start at zero.

A simulation of the electric field on the switch center plane using a custom electrostatic relaxation solver is shown in Figure 7.
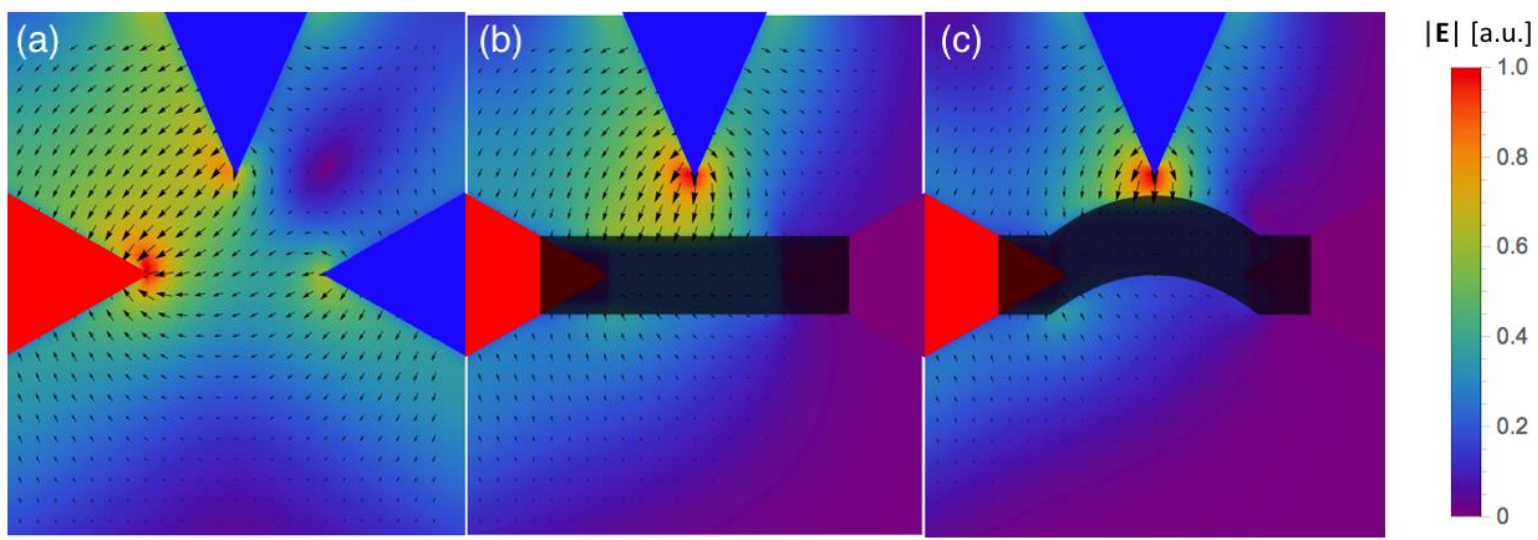

Figure 7. Electric fields in the switch: (a) at the initial state, (b) when preliminary arc strikes, and (c) after arc deflection. Electrode potential is color-coded with red $>$ purple $>$ blue. 
A simplified model for the transverse position of the arc center point was employed:

$$
y=y_{0}(V)+\kappa|\boldsymbol{J} \times \boldsymbol{B}| t^{2}
$$

where $y_{0}(V)$ is a function, which describes the initial position of the arc based on the voltage of the electrodes, and $\kappa$ is a normalization factor to convert the magnetic force to an acceleration. By using a first-order approximation for the position function $y_{0}(V) \approx y_{0}+y_{1} V$ and approximating the magnetic force as proportional to voltage, i.e., $|\boldsymbol{J} \times \boldsymbol{B}| \propto V$, Equation (1) was simplified as:

$$
y=y_{0}+y_{1} V+\kappa V t^{2}
$$

The first-order approximation has three free variables to fit: $y_{0}, y_{1}, k$. Figure 8 shows the best-fit result. Note the deviation from this behavior for the smallest shunt spacing. In this case, the fit was poor and has been omitted. For such a small spacing, the handoff takes place in only a few microseconds: less than two full periods. In such a scenario, the approximation of a constant force is not valid; instead, the more relevant metric is the phase within the RLC ringing, leading to this flattening of the slope.

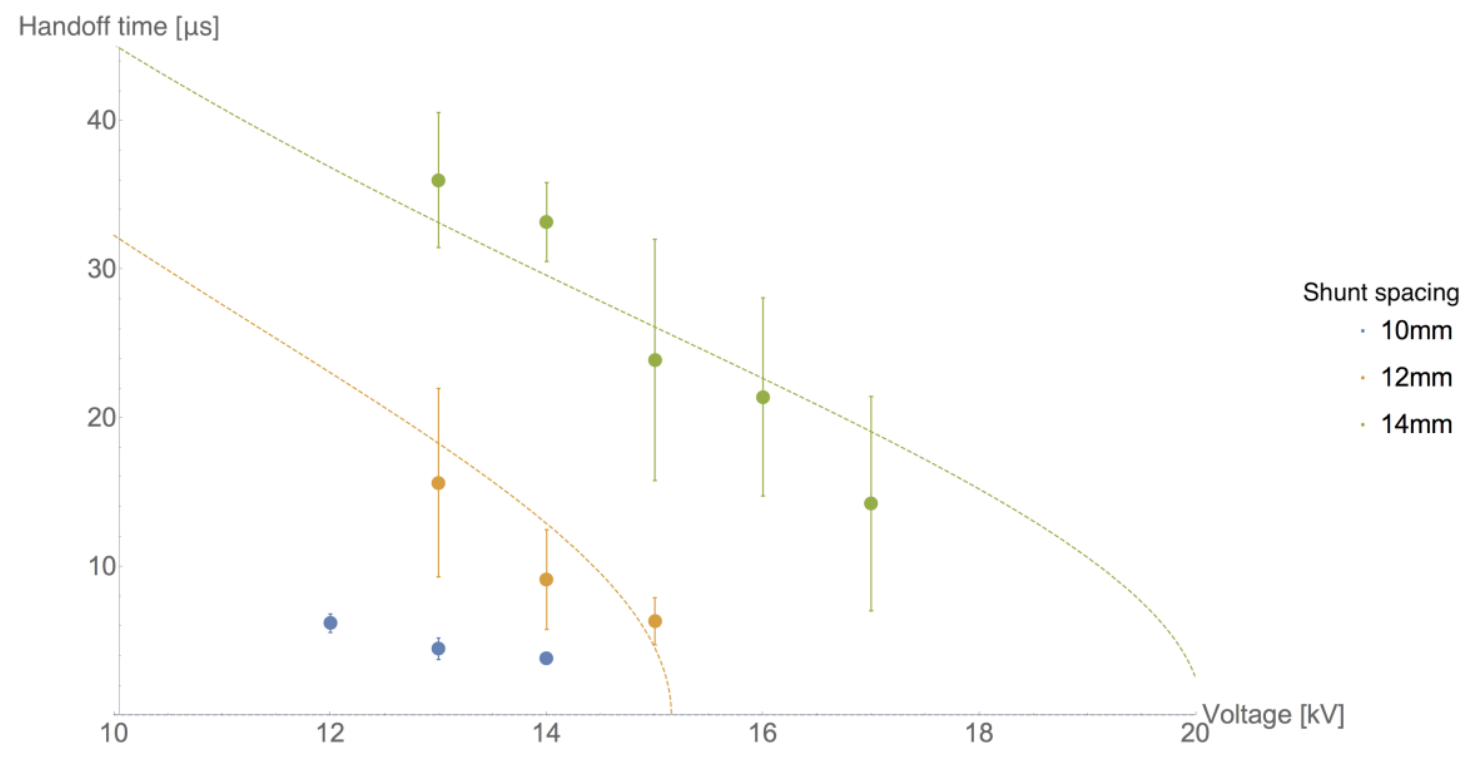

Figure 8. Time of current handoffs for different voltages and shunt spacings.

\section{Discussion}

We have demonstrated a new type of plasma opening switch, capable of switching on and off kiloamp currents at kilovolt potentials on microsecond timescales. We envisaged two parallel routes forward. First, our immediate interest in this device is it could be used as a way to reduce the thermal load on a small, high-repetition-rate, dense plasma focus (DPF) [10]. By redirecting the flow of current after the first period, the DPF dynamics-important application metrics, such as neutron and X-ray production-were unaffected, but the thermal load on the device was reduced by up to $90 \%$. Secondly, we also intended to further probe the inner workings of this device by using ultrafast laser and framing camera diagnostics [10]. This will require replacing the neodymium magnets with pulsed coils, as illustrated in Figure 9. 


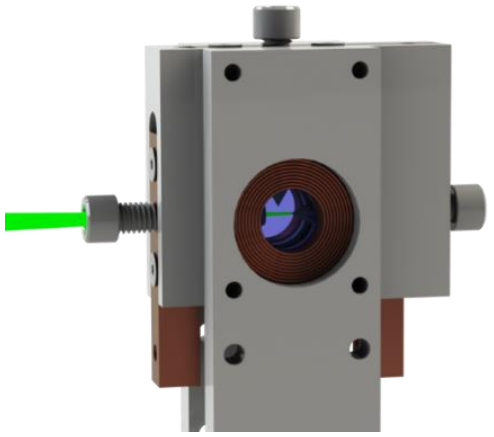

Figure 9. A proposed future version of the switch with pulsed electromagnets to allow for optical diagnostics.

Author Contributions: Conceptualization, J.R. and N.M.; Methodology, N.M. and W.L.; Software, N.M.; Validation, N.M., W.L., Y.S., J.R.; Formal Analysis, N.M.; Investigation, N.M. and W.L.; Resources, J.R. and Y.S.; Writing-Original Draft Preparation, N.M.; Writing-Review \& Editing, N.M., W.L., Y.S., J.R.; Visualization, N.M.; Supervision, J.R. and Y.S.; Project Administration, J.R. and N.M.; Funding Acquisition, J.R.

Funding: This work was funded by the Defense Advanced Research Projects Agency (DARPA) program for Intense and Compact Neutron Sources (ICONS), award number HR0011-15-2-0013.

Conflicts of Interest: The authors declare no conflicts of interest.

\section{References}

1. Tesla, N. Experiments with Alternate Currents of High. Potential and High. Frequency; Institution of Electrical Engineers: New York, NY, USA; London, UK, 1892.

2. Kloneck, A. Rotary Spark-Gap. U.S. Patent 1,366,160, 18 January 1921.

3. Fowler, R.G.; Wolfe, R.A. A Non-Mechanical Interrupter for Controlled Spark Circuits. J. Opt. Soc. Am. 1945, 35, 170-174. [CrossRef]

4. Armor, J. Method of Producing High-Frequency Oscillatory Currents. U.S. Patent 1,216,646, 14 October 1911.

5. Savage, M.E.; Hong, E.R.; Simpson, W.W.; Usher, M.A. Plasma Opening Switch Experiments at Sandia National Laboratories. In Proceedings of the 10th International Conference on High-Power Particle Beams, San Diego, CA, USA, 20-24 June 1994; pp. 41-44.

6. Meger, R.A.; Commisso, R.J.; Cooperstein, G.; Goldstein, S.A. Vacuum inductive store/pulse compression experiments on a high power accelerator using plasma opening switches. Appl. Phys. Lett. 1983, 42, 943-945. [CrossRef]

7. Bluhm, H. Pulsed Power Systems; Springer: Berlin/Heidelberg, Germany, 2006.

8. Schoenbach, K.H.; Kristiansen, M.; Schaefer, G. A Review of Opening Switch Technology for Inductive Energy Storage. Proc. IEEE 1984, 72, 1019-1040. [CrossRef]

9. Mendel, C.W.; Savage, M.E.; Zagar, D.M.; Simpson, W.W.; Grasser, T.W.; Quintenz, J.P. Experiments on a current-toggled plasma-opening switch. J. Appl. Phys. 1992, 71, 3731-3746. [CrossRef]

10. Majernik, N.; Pree, S.; Sakai, Y.; Naranjo, B.; Putterman, S.; Rosenzweig, J.B. Simultaneous Ultra-Fast Imaging and Neutron Emission from a Compact Dense Plasma Focus Fusion Device. Instruments 2018, 2, 6. [CrossRef]

(C) 2018 by the authors. Licensee MDPI, Basel, Switzerland. This article is an open access article distributed under the terms and conditions of the Creative Commons Attribution (CC BY) license (http:/ / creativecommons.org/licenses/by/4.0/). 